\title{
Flip-flop modulation method used with a pyramid wavefront sensor to correct piston segmentation on ELTs
}

\author{
Byron Engler, ${ }^{\mathrm{a}, \mathrm{b}, *}$ Miska Le Louarn $\odot{ }^{\mathrm{b}}{ }^{\text {Christophe Vérinaud, }}{ }^{\mathrm{b}}$ \\ Steve Weddell $\odot,{ }^{a}$ and Richard Clare ${ }^{\mathrm{a}}$ \\ ${ }^{a}$ University of Canterbury, Department of Electrical and Computer Engineering, Christchurch, \\ New Zealand \\ ${ }^{\mathrm{b}}$ European Southern Observatory, Garching bei Muenchen, Germany
}

\begin{abstract}
The next generation of ground-based optical telescopes, such as the European Southern Observatory's Extremely Large Telescope (the ELT), will have large support structures (spiders) for the secondary mirror. These spiders have the effect of segmenting the pupil. Without careful control of the wavefront, segment piston (petal modes) errors can develop. We present a flip-flop modulated/unmodulated method for the pyramid wavefront sensor (PWFS) enabling the PWFS to sense petal piston modes. This flip-flop modulation method uses a single PWFS operating in two states: a modulated state and an unmodulated state. An independent controller is used in each state; the modulated state controls the atmospheric turbulence and the unmodulated state only controls petal piston modes. In simulation, we show the flip-flop method working with the wavefront sensor in both K- and R-bands, providing an improvement of $9.9 \%$ and $13 \%$, respectively, over a standard modulated PWFS. $\odot$ The Authors. Published by SPIE under a Creative Commons Attribution 4.0 International License. Distribution or reproduction of this work in whole or in part requires full attribution of the original publication, including its DOI. [DOI: 10 .1117/1.JATIS.8.2.021502]
\end{abstract}

Keywords: pyramid wavefront sensor; adaptive optics; segment piston.

Paper 21114SS received Sep. 7, 2021; accepted for publication Dec. 20, 2021; published online Mar. 2, 2022.

\section{Introduction}

The next generation of ground-based astronomical telescopes and associated instrumentation are currently under development. These so-called extremely large telescopes (ELTs) comprise the European Southern Observatory's (ESO) Extremely Large Telescope (the ELT), ${ }^{1}$ the Giant Magellan Telescope, ${ }^{2}$ and the Thirty Meter Telescope. ${ }^{3}$ These ELTs will have primary mirrors of diameter on the order of 25 to $37 \mathrm{~m}$.

Earth's time-varying turbulent atmosphere limits the achievable resolution of ground-based telescopes. Adaptive optics (AO) systems are used to measure and correct the blurring effects of the turbulence in real time using a wavefront sensor (WFS) to measure wavefront errors caused by the atmosphere and a deformable mirror (DM) to correct for them. ${ }^{4,5}$

A critical component of any AO system is the WFS. The measurements from the WFS are used to estimate the phase of the incoming wavefront. In current AO systems, the Shack-Hartmann WFS (SHWFS) is commonly used. The ELTs will predominantly use a relatively new WFS known as the pyramid WFS (PWFS) for single-conjugate AO. ${ }^{6}$ The PWFS offers improved sensitivity, in terms of limiting magnitude, in closed-loop, over the SHWFS. ${ }^{7-9}$ The PWFS, shown in Fig. 1, consists of a glass pyramid, which is placed at the focal point of the telescope such that the focal point falls on the tip of the pyramid. The pupil plane is then reimaged, via relay optics, to the detector. The resulting detector plane consists of four pupil images, which are used to estimate the wavefront. The PWFS has a small dynamic range, when compared with an SHWFS, because the sensor is only in the linear region when the focal spot is on the apex of the pyramid. A method to overcome the limited dynamic range is to modulate the

*Address all correspondence to Byron Engler, byron.engler@eso.org 


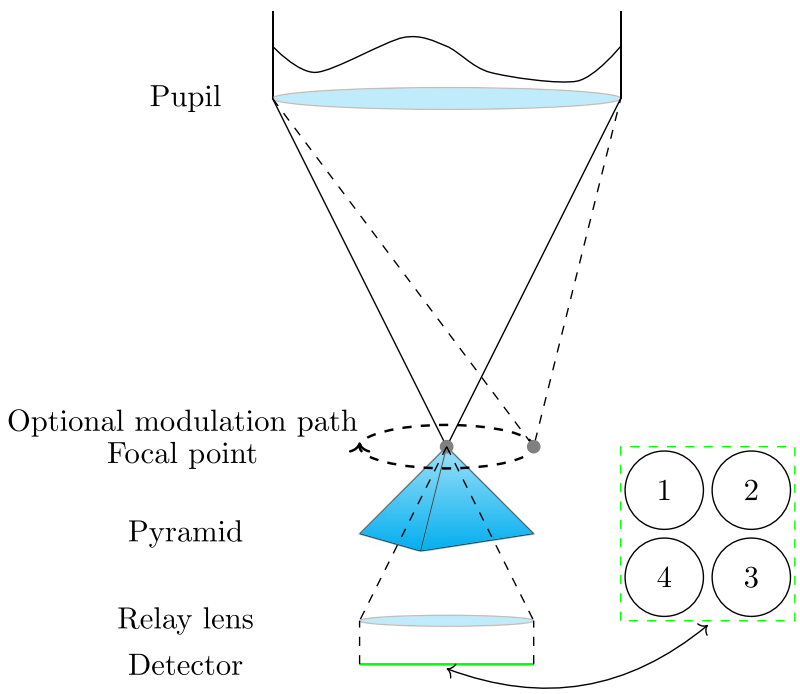

Fig. 1 Schematic of the pyramid WFS. A four-sided prism (pyramid) is placed in the focal plane of the telescope. Starlight travels through the atmosphere and becomes distorted. The pupil focuses the light onto the apex of the pyramid. The pyramid subdivides the focal plane into quadrants. The output from the prism is focused onto a detector by the relay lens, forming four images of the pupil. The focal point can be modulated around the apex of the pyramid, as shown by the dotted circle, to improve the dynamic range and linearity.

focal point around the tip of the pyramid and the detector image is integrated over the modulation path. ${ }^{10}$ This modulation trades sensitivity for dynamic range.

The measurement vector, $s$, of a PWFS is expressed as

$$
s=P(\phi)-P\left(\phi_{\mathrm{ref}}\right)
$$

where $P$ is the pyramid operator, $\phi$ is the phase of the atmospheric wavefront, and $\phi_{\text {ref }}$ is a flat reference (zero) wavefront. The measurement is a linear combination of the four pupil images, ${ }^{6}$ often referred to as the $x$ and $y$ slope maps ( $s_{x}$ and $s_{y}$, respectively). The slope maps used in this paper are defined as

$$
\begin{aligned}
& s_{x}=\frac{I_{1}(x, y)-I_{2}(x, y)-I_{3}(x, y)+I_{4}(x, y)}{\sum_{i=1}^{4} I_{i}(x, y)}, \\
& s_{y}=\frac{I_{1}(x, y)+I_{2}(x, y)-I_{3}(x, y)-I_{4}(x, y)}{\sum_{i=1}^{4} I_{i}(x, y)} .
\end{aligned}
$$

Due to the ELTs' physical size, the support structure (or spider) that holds the secondary mirror in place is also large. In the case of the ESO's he ELT, the spider has six support beams with a thickness of $0.5 \mathrm{~m}$. ${ }^{11}$ These spider arms have the effect of dividing the pupil into six segments (or petals), which is shown in Fig. 2. The width of the spider arms is greater than the typical coherence length of the atmosphere, or Fried parameter $r_{0}$, expected at the observatory sites. This introduces complexities in the wavefront estimation, as there is no phase continuity between the six pupil segments, as viewed from the WFS. Consequently, the average wavefront over each pupil segment (segment piston) can be different, and a modulated pyramid WFS is not very sensitive to these segment piston modes.

Figure 3 shows the simulated residual optical path difference over the ELT pupil with the WFS operating in K-band without petal mode corrections. The resulting closed-loop Strehl is $\sim 42 \%$, compared with $96 \%$ without the spider and associated segment piston errors. With the WFS in R-band, we are able to close the loop, but with poor performance resulting from uncorrected segment piston errors. 

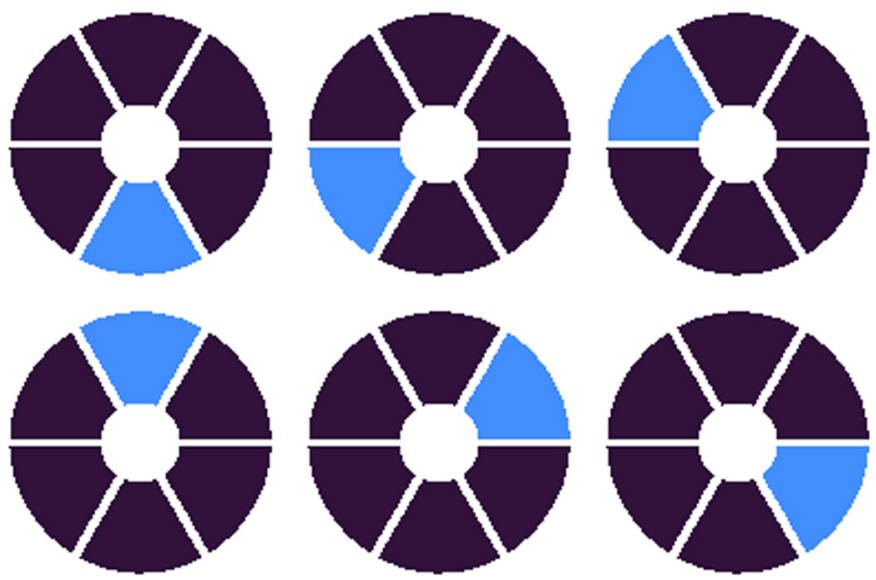

Fig. 2 The six segment piston modes of the ELT.

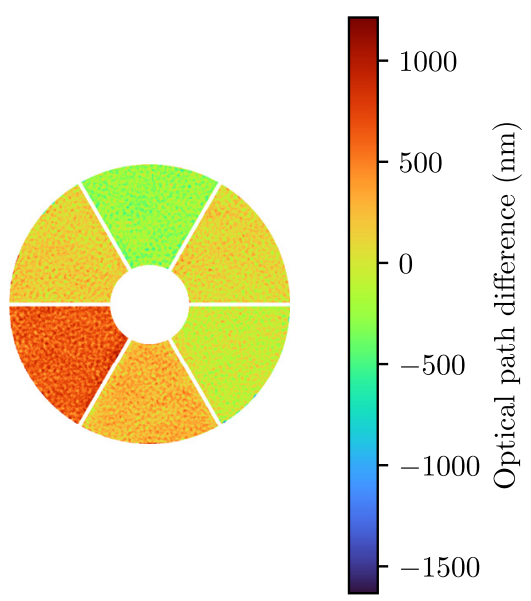

Fig. 3 The residual optical path difference with an atmosphere with $r_{0}$ of $15 \mathrm{~cm}$, using a modulated pyramid wavefront sensor and making no special effort to correct the segment piston errors.

The main contributing factors to the segment piston problem are the so-called low wind effect, segment piston due to atmospheric turbulence, segment piston due to modal aliasing of the modal basis functions used for wavefront control, and poor sensitivity to segment piston modes. The low wind effect is a phenomenon where the low-velocity wind blowing over the telescope structure is cooled to subambient temperatures, resulting in optical path differences across the pupil of the telescope. ${ }^{12}$

Previous work on the topic of segment piston control has led to several approaches to solving the problem. Schwartz et al. ${ }^{11}$ shown a phase closure algorithm to ensure a continuous phase across pupil segments. Their algorithm uses the amplitude of actuator commands along adjacent pupil segment edges to estimate the correction phase required to ensure continuous phase across pupil segments. The authors also suggest that the pupil segment piston modes can be filtered from the modal basis. However, the AO system would then be unable to correct the atmospheric segment piston modes. Finally, they show that by slaving adjacent actuators across pupil segments, the DM behaves as if the surface were continuous, therefore eliminating segment piston errors.

Hutterer et al. ${ }^{13}$ proposed a method using a pyramid WFS with a split controller approach, where two reconstructors are used. In their split approach, one reconstructor uses a segment piston-free modal basis and corrects for the main part of the atmospheric turbulence. A second reconstructor is used only to reconstruct the segment piston modes. The resulting command for the DM is the combination of both reconstructors. They show this method working with a modulated pyramid WFS operating in K-band only. 
Bertrou-Cantou et al. ${ }^{14}$ proposed a continuous basis, where the first-order derivative of each mode across the spider is forced to be continuous (the gradient on both sides of the spider are equal). The continuous basis is also formed in such a way as to follow Kolmogorov statistics. A linear combination of modes from the continuous basis cannot result in pure segment piston modes; however, segment piston modes can be generated in combination with other modes (e.g., astigmatism). The continuous basis has been shown to perform better than actuator slaving and significantly improves on the standard Karhunen-Loève (KL) basis.

In previous work, ${ }^{15}$ we have shown that by reducing the number of modes in the modal basis, the petal effect can be reduced by limiting modal aliasing of higher-order modes. This method will not help in correcting atmospheric or telescope-induced petal piston errors.

Esposito et al. ${ }^{16}$ shown that with a reduced number of modes in the modal basis, careful filtering of modes during the interaction matrix inversion, and by applying a mask to the WFS measurements that selects subapertures that are sensitive to segment piston, they are able to control segment piston modes. However, the segments occasionally jump between integer multiples of the sensing wavelength.

In Ref. 17, we show that an unmodulated PWFS is significantly more sensitive to petal piston than a modulated PWFS. In this paper, we propose a flip-flop modulation scheme where a single pyramid is used, and the modulation alternates between modulated and unmodulated. The flipflop modulation method gives the benefits of increased dynamic range (modulated pyramid), useful for closing the loop, and increased sensitivity to segment piston modes (unmodulated pyramid). Previously, we have shown that within a $100-\mathrm{Hz}$ bandwidth, greater than $95 \%$ of atmospheric petal modes can be corrected with a pseudo-open-loop flip-flop modulation PWFS ${ }^{17}$ Here, we show that with careful selection of the modal basis and calibration, it is possible to sense and control petal piston modes, in the presence of closed-loop AO residuals, within an amplitude range of one wavefront sensing wavelength.

The PWFS has been shown to be nonlinear. ${ }^{7}$ Typically, a linear reconstruction model is used, which has been calibrated around some operating point. For the PWFS, the calibration is done in a diffraction-limited regime, while the wavefront sensing is done in the presence of atmospheric turbulence and AO loop residuals. It has been shown that a modal gain correction ${ }^{18-20}$ (optical gain) is necessary to move the diffraction-limited model to the expected operating point.

The paper is structured as follows. In Secs. 2.1 and 2.2, we describe the modal basis used for our two control loops. Section 2.3 outlines the optical gain corrections needed for each modal basis. In Sec. 2.4, we introduce an optical gain of the pyramid reference signal. Sections 3.1 and 3.2 show the simulation results of the flip-flop method, with the WFS in K-band and R-band, respectively. We summarize our approach to the problem and outline areas for future work in Sec. 4.

\section{Methodology}

In this paper, the closed-loop flip-flop modulation method is presented in simulation. Figure 4 shows a block diagram of the flip-flop modulation scheme, where two separate reconstructors are used. One reconstructor corrects the bulk of the atmospheric turbulence, using a KL basis. The other reconstructor corrects for segment piston modes only and consists of the segment piston eigenmodes (excluding pure piston). The segment piston reconstructor takes measurements from an unmodulated pyramid, whereas the atmospheric reconstructor takes measurements from a modulated pyramid. Using a single pyramid and switching between modulated and unmodulated states, both reconstructors use the same optical hardware. In simulation, a dead-time of one frame is used to allow the modulator to stop and to restart. For this paper, 10 frames are run with the modulated pyramid, then one frame is discarded, one frame of the unmodulated pyramid is captured, then another frame is discarded. The sequence is repeated. The dead frame is there to account for real-life effects (for example, the modulator not being able to accelerate at an infinite rate.) A similar control structure could be implemented with separate modulated and unmodulated pyramids, removing the need to stop the modulation, but would require splitting the light. 


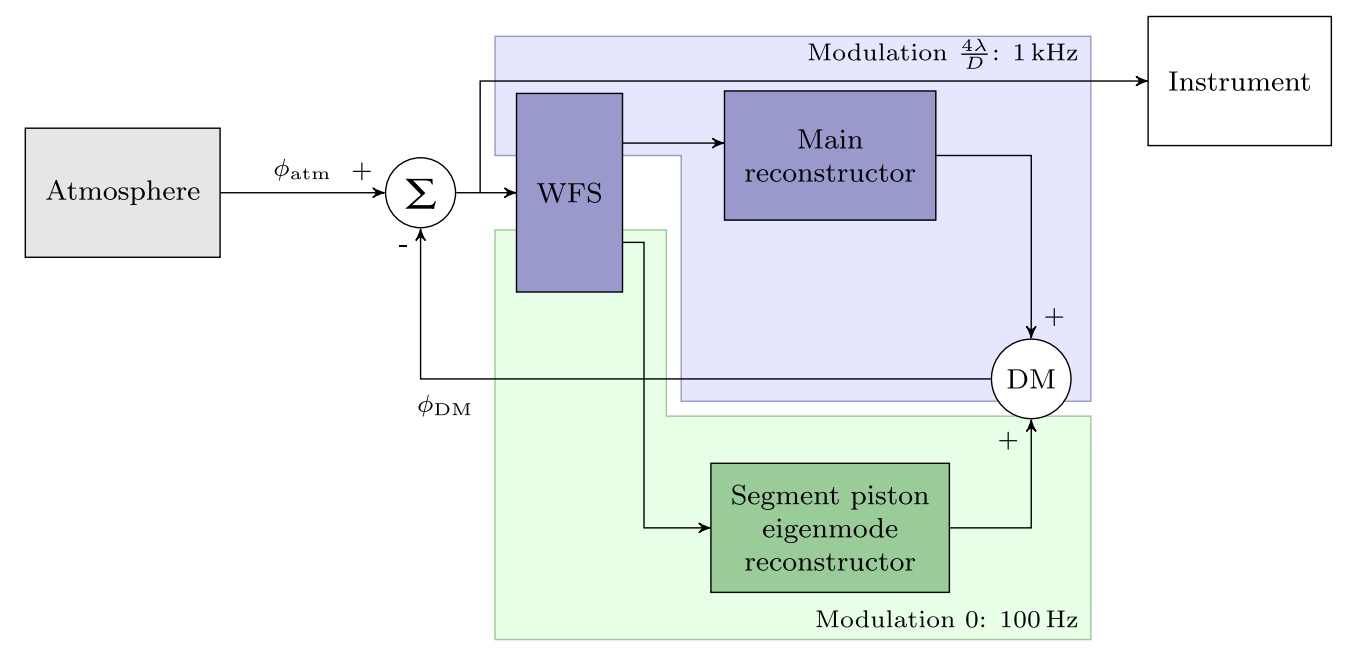

Fig. 4 The flip-flop modulation pyramid WFS. Two AO control loops operate with a single pyramid. The modulation and associated control loop is switched on and off, allowing the increased dynamic range of a modulated pyramid (blue) for correcting the majority of the atmospheric turbulence and the improved sensitivity of an unmodulated pyramid WFS (green) to control the segment piston modes.

Using the following steps, it is possible to control segment piston errors with a single pyramid WFS:

1. Find a modal basis for correcting the segment piston modes.

2. Find a modal basis for correcting the atmospheric turbulence.

3. Find the optical gains of the segment piston modal basis, for an unmodulated pyramid.

4. Find the optical gains of the atmospheric modal basis, for a modulated pyramid.

5. Build reconstructors for both modulated and unmodulated loops, with optical gain compensation.

6. Run the AO system and correct segment piston errors.

\subsection{Finding a Modal Basis for the Unmodulated Pyramid}

The choice of modal basis is a critical step in controlling the petal piston modes. First, the system response to the segment piston modes is recorded in an interaction matrix, $F_{\mathrm{sp}}$, for a diffractionlimited system. The interaction matrix is generated by applying each segment piston mode, as shown in Fig. 2, to the DM. The resulting wavefront is then propagated to the pyramid WFS, and the wavefront measurement is recorded. Each measurement is a row in the interaction matrix, $F_{\text {sp }}$. Then, using singular value decomposition, the segment piston interaction matrix, $F_{\mathrm{sp}}$, is decomposed into singular values (or eigenmodes), which are a linear combination of individual segment piston modes:

$$
F_{\text {sp }}=U S V^{T},
$$

where $U$ and $V$ are the unitary matrices and $S$ is a diagonal matrix of singular values (square root of the eigenvalues). $V^{T}$ are the eigenvectors of $F_{\text {sp }}$. From this factorization, the segment piston eigenmode basis is constructed as

$$
Z_{\text {eigen }}(i)=\sum_{m=1}^{M} V_{i, m} Z_{\mathrm{sp}}(m)
$$

where $Z_{\mathrm{sp}}$ is the modes-to-command matrix for the segment piston modes as shown in Fig. 2, $Z_{\text {eigen }}$ is the modes-to-command matrix (DM commands for each mode) for the segment piston eigenmodes, $i$ is the eigenmode index, and $M$ is the number of segment piston modes. The 

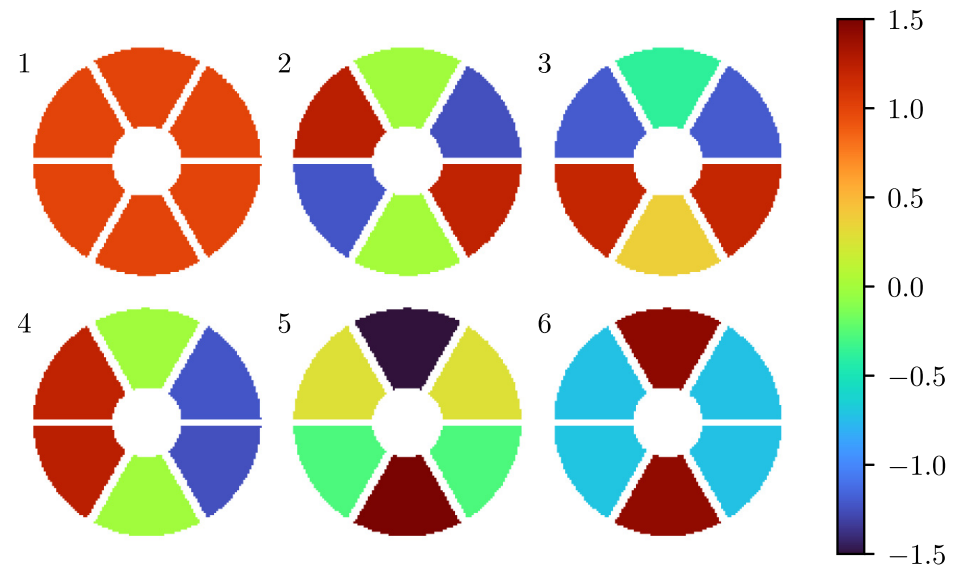

Fig. 5 The segment piston eigenmodes, including piston, for an unmodulated pyramid WFS. The modes are ordered from least sensitive to most sensitive (top left to bottom right). The amplitude of each mode has been normalized such that the RMS over the pupil is one.

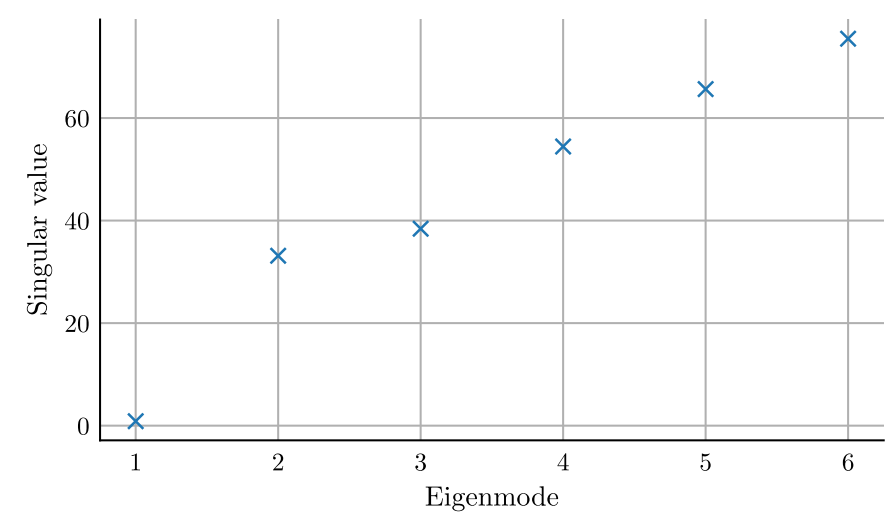

Fig. 6 The segment piston eigenvalues, where mode one corresponds to pure piston with a singular value of near zero. The mode ordering is the same as Fig. 5.

resulting segment piston eigenmodes, $Z_{\text {eigen }}$, are shown in Fig. 5 (unmodulated case) and the corresponding segment piston singular values are shown in Fig. 6. The higher the singular value, the more sensitive the WFS is to the corresponding eigenmode.

In this paper, the unmodulated PWFS is only used to control petal modes. In future work, the number of modes controlled by the unmodulated PWFS could be increased.

\subsection{Finding a Modal Basis for the Modulated Pyramid}

The modulated pyramid WFS is used to control the bulk of the atmospheric turbulence, due to the increased dynamic range from the modulation. This enables the AO system to reliably close the loop. To produce an efficient reconstructor, it is necessary to have an orthogonal modal basis. The modal basis is orthogonalized twice: once with respect to the actuator geometry and a second time with respect to the atmospheric covariance. This process of double diagonalization is described in Refs. 21 and 22. A key step is the computation of the atmospheric covariance, $\left\langle h_{i} h_{j}\right\rangle$, which is defined as

$$
\left\langle h_{i} h_{j}\right\rangle=\operatorname{Re}\left[\sum_{u=0}^{P-1} \sum_{v=0}^{Q-1}\left(\mathcal{F}\left[\operatorname{IF}_{i}(x, y)\right]^{*} \cdot \mathcal{F}\left[\operatorname{IF}_{j}(x, y)\right] \cdot \operatorname{PSD}_{\phi}(u, v) \cdot d f^{2}\right)\right],
$$

where $\mathrm{PSD}_{\phi}$ is the power spectral density of the atmospheric turbulence, IF is a single influence function of the DM (as shown in Fig. 7), $d f$ is the frequency step size used in $\mathrm{PSD}_{\phi}, P$ and $Q$ are 


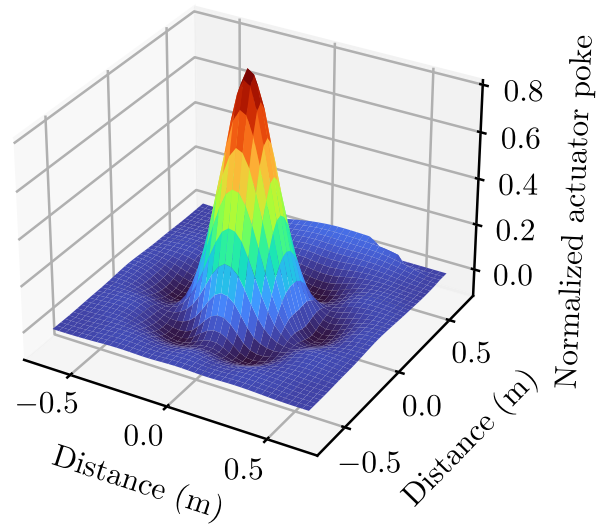

Fig. 7 An influence function of the ELT DM (M4), derived from finite element analysis and interpolated to the primary mirror space (M1).

the number of elements in the $u$ and $v$ axes, respectively, and $*$ denotes the complex conjugate. The statistical covariance matrix $\Delta_{\text {stat }}$ is defined as

$$
\Delta_{\text {stat }}=\left[\begin{array}{cccc}
\left\langle h_{1} h_{1}\right\rangle & \left\langle h_{1} h_{2}\right\rangle & \ldots & \left\langle h_{1} h_{j}\right\rangle \\
\vdots & \ddots & & \vdots \\
\left\langle h_{i} h_{1}\right\rangle & \ldots & & \left\langle h_{i} h_{j}\right\rangle
\end{array}\right] .
$$

It is useful to be able to force certain modes to be present (initially these will be piston, tip and tilt), regardless of the double orthogonalization steps. For example, the tip and tilt can be offloaded to a separate control system. For each actuator in the DM, the influence function is flattened into a row vector. The influence function row vectors are stacked into a matrix IF, where the row is indexed by the actuator number. The first 20 modes, excluding piston, for a KL modal basis that is forced orthogonal to piston, tip, and tilt are shown in Fig. 8.

A modal basis, which is forced to be orthogonal to the segment piston eigenmodes, is also needed. If the segment piston eigenmodes are present in the modal basis used by the modulated pyramid WFS, the two controllers (modulated and unmodulated) will contest each other. The
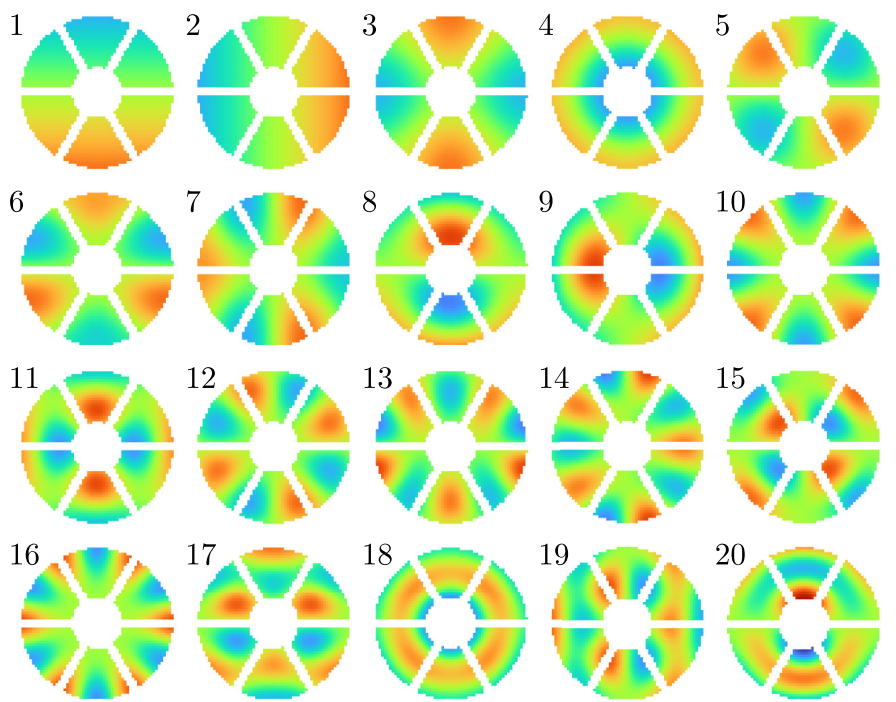

20
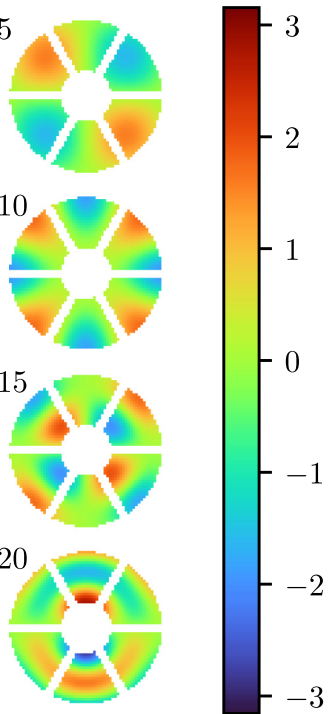

Fig. 8 The first $20 \mathrm{KL}$ modes (excluding piston), forced to be orthogonal to piston, tip, and tilt, which are produced using the double orthogonalization method, without forcing orthogonality to the six petal eigenmodes (normalized to an RMS of $1 \mathrm{~nm}$ ). 


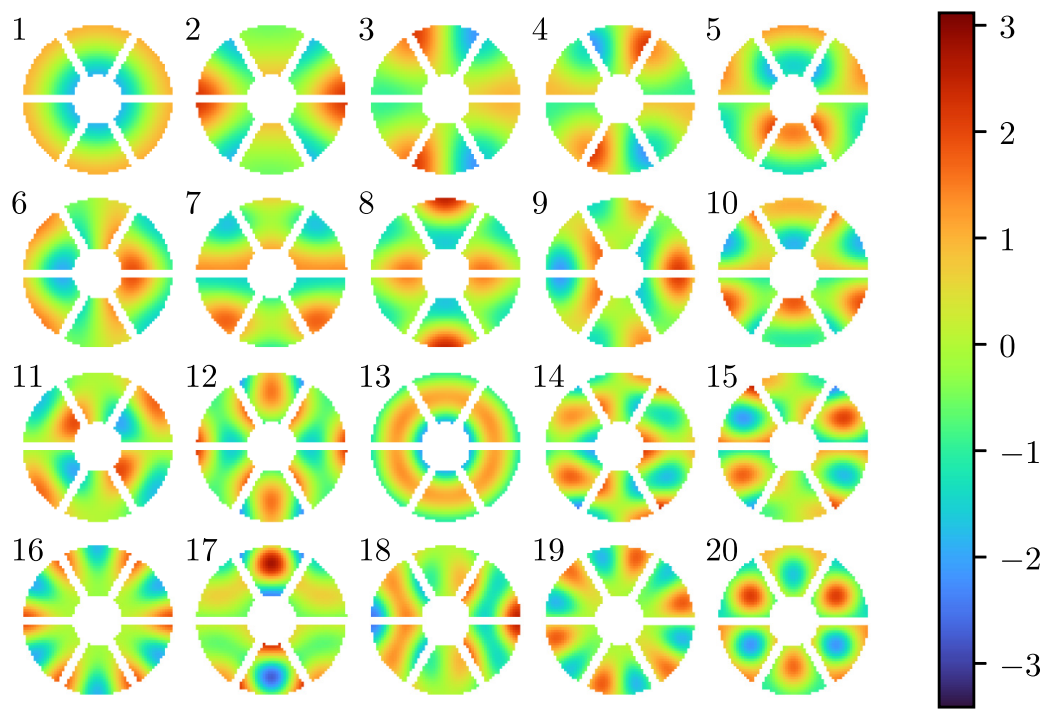

Fig. 9 The first $20 \mathrm{KL}$ modes (excluding piston), forced to be orthogonal to the six segment piston eigenmodes, which are produced using the double orthogonalization method.

first 20 segment piston-free modes are shown in Fig. 9. In the segment piston-free modal basis, tip and tilt modes are not constrained and exist throughout the basis (the modal basis is not forced to be orthogonal to tip and tilt).

\subsection{Optical Gain Compensation for the Pyramid}

Now that a modal basis for each control loop has been defined, a reconstructor that accurately reconstructs a wavefront from a pyramid WFS measurement is required. The pyramid WFS frequency response is not linear, ${ }^{18-20}$ especially in the presence of $\mathrm{AO}$ residual. The effective gain for each mode is not unity. This phenomenon is known as the optical gain problem. To solve the optical gain problem, the effective gain for each mode needs to be found. Interaction matrices capturing the response of the pyramid WFS to each mode in the basis are measured for both a diffraction-limited case, and with AO residual present. The AO residual fitting error, $\phi_{\mathrm{fit}}$, is found by subtracting the projection of each mode in the basis from an atmospheric phase screen, $\phi_{\mathrm{fit}}$,

$$
\phi_{\mathrm{fit}}(x, y)=\phi_{\mathrm{atm}}(x, y)-\sum_{i=1}^{N} \phi_{\mathrm{atm}}(x, y) \cdot \phi_{Z_{i}}(x, y),
$$

where $N$ is the number of modes in the modal basis, and $\phi_{Z_{i}}(x, y)$ is the phase of the $i$ 'th mode in the modal basis. The DM command, $\phi_{\mathrm{DM}}$, is calculated as follows:

$$
\phi_{\mathrm{DM}}=B s,
$$

where $B$ is the modal command matrix which has optical gain compensation applied and $s$ is the measurement vector from the pyramid WFS. $B$ is defined as

$$
B=Z \cdot\left(\frac{1}{O G} \cdot F^{\dagger}\right)
$$

where $Z$ is the modes-to-command matrix, $F$ is the modal interaction matrix, and $\dagger$ denotes the pseudoinverse. The optical gains, OG, are defined as $^{20}$

$$
\mathrm{OG}=\frac{\operatorname{diag}\left(F_{\text {res }}^{T} \cdot F_{\text {diff }}\right)}{\operatorname{diag}\left(F_{\text {diff }}^{T} \cdot F_{\text {diff }}\right)},
$$




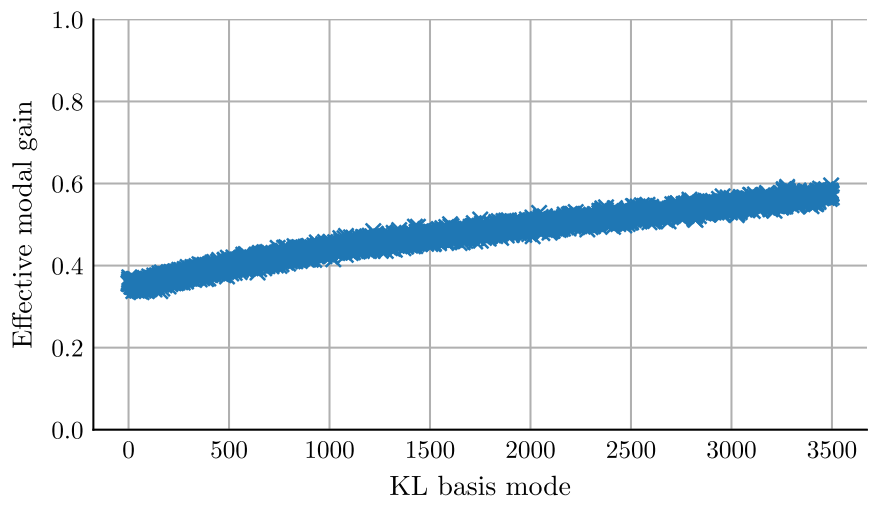

Fig. 10 The optical gains of the segment piston-free KL modes (Fig. 9) for a modulated pyramid WFS operating in R-band, using the default parameters from Table 1.

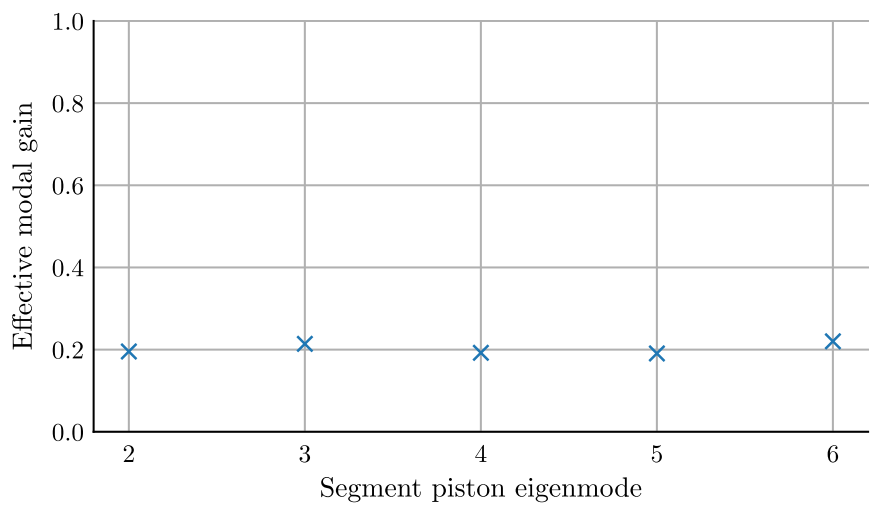

Fig. 11 The optical gains of the segment piston eigenmodes for an unmodulated pyramid WFS operating in R-band, using the default parameters from Table 1.

where $F_{\text {res }}$ is the interaction matrix in the presence of AO residual, and $F_{\text {diff }}$ is the diffractionlimited interaction matrix. The optical gains are calculated for both the modulated pyramid WFS with the entire modal basis, and the unmodulated pyramid WFS with only the segment piston eigenmodes. The optical gains for the modulated pyramid WFS are shown in Fig. 10, and the optical gains for the segment piston eigenmodes are shown in Fig. 11.

\subsection{Reference Measurement in the Presence of AO Residual}

When testing the unmodulated pyramid WFS reconstructor (with optical gain compensation), it is found that certain segment piston eigenmodes present an offset. The eigenmode offset is shown in Fig. 14, where a 50-nm poke for each eigenmode is presented to the unmodulated pyramid WFS. In the ideal case, the reconstructed wavefront should match the eigenmode poke, as is the case for eigenmodes one through three, where a $50 \mathrm{~nm}$ poke of each eigenmode is reconstructed with negligible modal crosstalk. For a 50-nm poke of eigenmode five, however, only $30 \mathrm{~nm}$ is reconstructed, as well as -35 to $-30 \mathrm{~nm}$ of the other four eigenmodes.

The standard method, Eq. (1), of forming a measurement from a pyramid WFS consists of subtracting a null reference measurement from the turbulence measurement. In the case of the diffraction-limited unmodulated pyramid WFS, the null reference measurement, as shown in Fig. 12, has a significantly different average value than that of the reference measurement measured in the presence of AO residual, as shown in Fig. 13. Due to the reduced amplitude of the null reference measurement, the traditional reference measurement subtraction overcompensates for the zero reference. Figure 14 also suggests that the measurement signal for eigenmode five is similar to the null reference. 


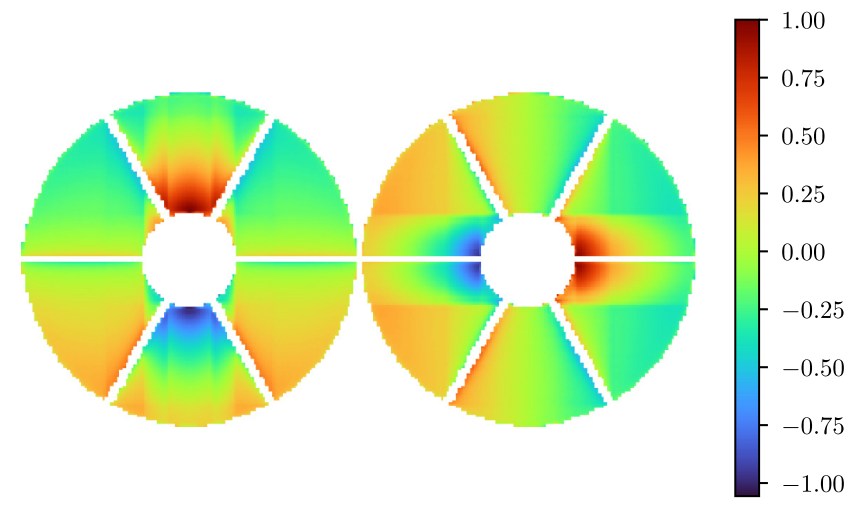

Fig. 12 The simulated slopes map (unitless) from an umodulated pyramid WFS, where the input wavefront is flat (zero across the entire pupil).

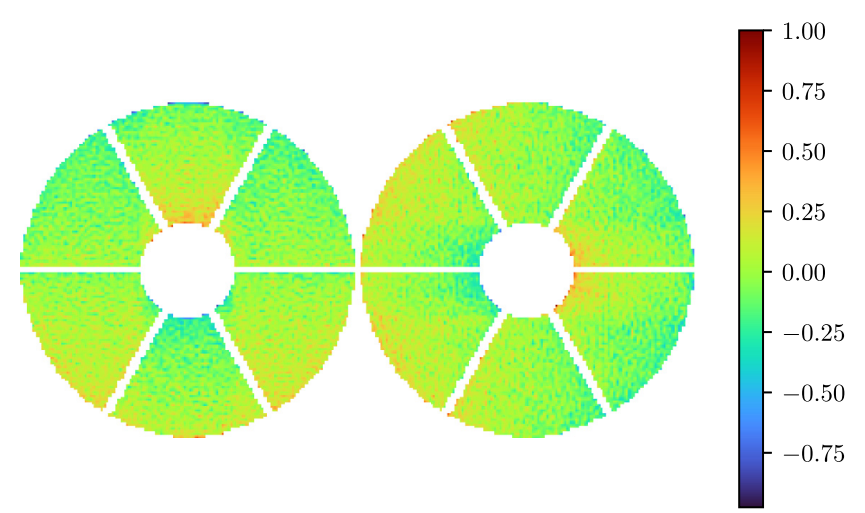

Fig. 13 The simulated slopes map (unitless) from an umodulated pyramid WFS, where the input wavefront is the residual fitting error obtained by removing the first 3500 modes from an atmospheric phase screen, averaged over 100 phase screens.

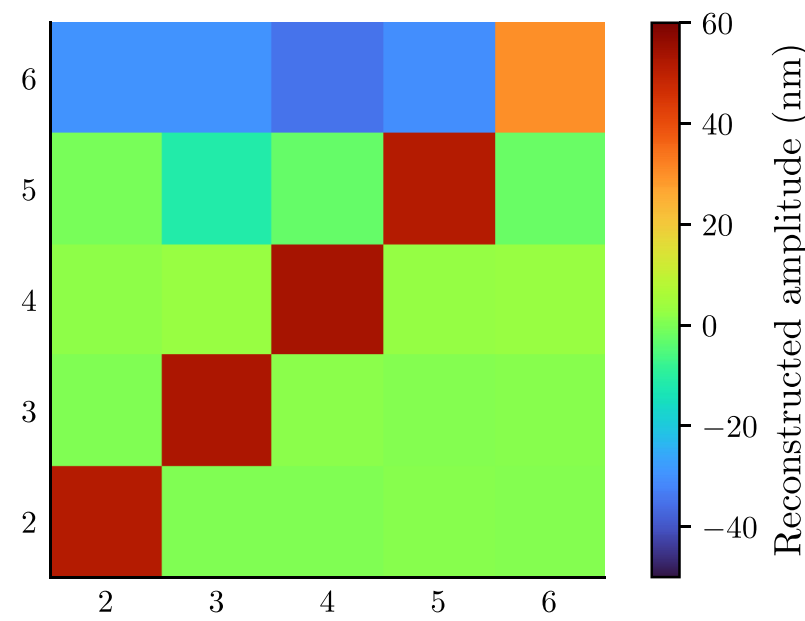

Fig. 14 The reconstructed amplitude for a 50-nm poke of each segment piston eigenmode in the presence of $A O$ residual. The reconstructor has had optical gain compensation applied to modes, and the standard reference measurement subtraction is applied. 


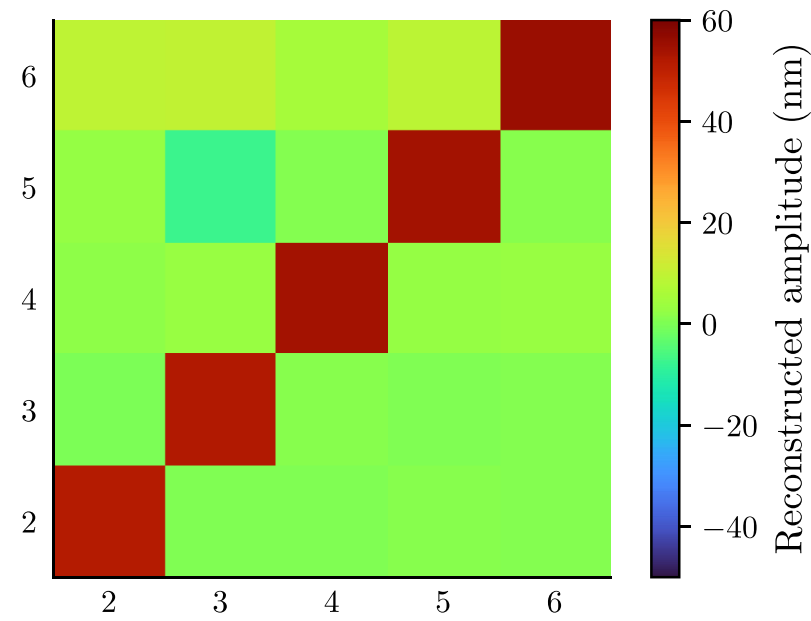

Fig. 15 The reconstructed amplitude for a 50-nm poke of each segment piston eigenmode in the presence of $\mathrm{AO}$ residual. The reconstructor has had optical gain compensation applied to modes, and no reference measurement is subtracted.

If instead, the reference measurement is not subtracted, the offset decreases and the segment piston reconstruction improves significantly, as shown in Fig. 15. The decrease in the offset is effectively an optical gain of the reference measurement and can be found via a similar process as that used for calculating modal optical gains. The reference measurement in the presence of AO residuals is effectively the diffraction-limited measurement scaled by a gain factor $k$

$$
s_{\text {ref }}=\frac{s_{\text {diff }}}{k},
$$

where $k$ is the optical gain of the reference signal, $s_{\text {diff }}$ is the diffraction-limited reference signal, and $s_{\text {ref }}$ is the optical gain compensated reference signal. $k$ is defined as ${ }^{19}$

$$
k=\frac{s_{\mathrm{res}}^{T} \cdot s_{\mathrm{diff}}}{s_{\mathrm{diff}}^{T} \cdot s_{\mathrm{diff}}},
$$

where $s_{\text {res }}$ is the pyramid WFS measurement signal in the presence of AO residuals. For the test case defined by Table $1, k$ is found to be $\sim 0.23$. This closely matches the optical gains for the segment piston eigenmodes in Fig. 11. By applying what is in essence an optical gain correction to the null reference measurement, the segment piston reconstruction is improved further. The segment piston reconstruction with optical gain compensation on both the reconstructor and null wavefront reference measurement are shown in Fig. 16. By applying the optical gain correction to the reference measurement, the offsets for certain segment piston eigenmodes are significantly reduced, and the cross-talk between modes is reduced.

\section{Simulation Results}

All of our simulations are performed using Octopus, ${ }^{23}$ an end-to-end simulation tool developed at ESO. The simulation parameters are listed in Table 1 and are used in all simulations except when stated otherwise. The pyramid is used without a field-stop (spatial filter). The active subaperture selection is based on an illumination threshold, where the illumination of subapertures with a spider is compared with the illumination without the spider. An illumination threshold of $50 \%$ was used, based on previous work. ${ }^{15}$ A nominal wavelength of 650 or $2200 \mathrm{~nm}$ is used for both the PSF and the WFS, in R- and K-bands, respectively. The main modulated PWFS AO loop is running at $1 \mathrm{kHz}$ and the segment piston correction loop with the unmodulated PWFS is effectively running at $100 \mathrm{~Hz}$ as the modulation is stopped every 10 iterations. In a real system, the tip/tilt mirror used to introduce the modulation is not able to stop or start the modulation instantly. To account for this in simulation, we insert a dead-frame before and after each single, 
Table 1 Simulation parameters used in this paper.

\begin{tabular}{|c|c|}
\hline Parameter & Value \\
\hline Telescope diameter $(D)$ & $37 \mathrm{~m}$ \\
\hline Secondary obstruction & $28 \%$ \\
\hline Fried parameter $\left(r_{0}\right)$ & $15 \mathrm{~cm}$ \\
\hline Outer scale $\left(L_{0}\right)$ & $25 \mathrm{~m}$ \\
\hline Atmosphere & ESO 35 layer model \\
\hline Frame rate $\left(F_{s}\right)$ & $1 \mathrm{kHz}$ \\
\hline Delay & Two frames \\
\hline Controller type & Integrator \\
\hline PSF $\left(\lambda_{p}\right)$ wavelength & $2200 \mathrm{~nm}$ (K-band) and $650 \mathrm{~nm}$ (R-band) \\
\hline WFS $\left(\lambda_{W}\right)$ wavelength & $2200 \mathrm{~nm}$ (K-band) and $650 \mathrm{~nm}$ (R-band) \\
\hline WFS order & $116 \times 116$ subapertures \\
\hline Modulation width & $4 \lambda_{W} / D,,^{10,24} 0 \lambda_{W} / D$ \\
\hline Modulation points & 32 \\
\hline Time steps & 10,000 \\
\hline Number of spider arms & 6 \\
\hline Spider arm width & $50 \mathrm{~cm}$ \\
\hline Flux & 10,000 photons/subaperture/frame \\
\hline Number of actuators & 5190 \\
\hline Number of modes & 3500 \\
\hline
\end{tabular}

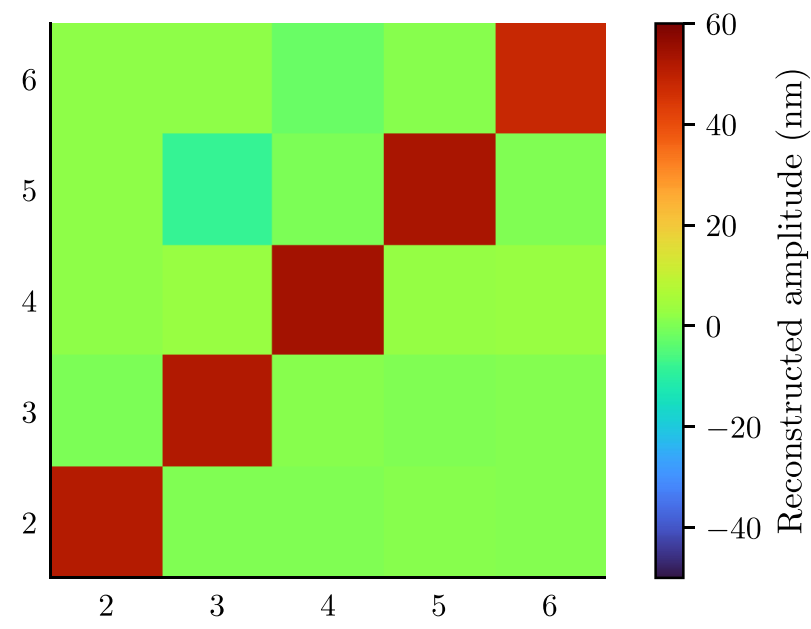

Fig. 16 The reconstructed amplitude for a 50-nm poke of each segment piston eigenmode in the presence of $A O$ residual. The reconstructor has had optical gain compensation applied to modes as well as the reference measurement. 
Table 2 Long exposure Strehl for R- and K-bands.

\begin{tabular}{lcc}
\hline \hline Band & Strehl with flip-flop modulation & Strehl with normal modulation \\
\hline $\mathrm{K}$ & $87.9 \%$ & $78.0 \%$ \\
$\mathrm{R}$ & $19.1 \%$ & $6.1 \%$ \\
\hline \hline
\end{tabular}

unmodulated PWFS measurement, where no WFS measurement is recorded. The long exposure Strehl is calculated after 30 iterations, when the AO loop is considered stable.

\subsection{K-Band Scenario}

In K-band, it is possible to close the loop either with or without the proposed flip-flop method. With the proposed flip-flop modulation method, an improvement in closed-loop long exposure Strehl of 9.9\% (Table 2) is observed over the Strehl of the standard modulated case. Figure 17 compares the short exposure Strehl, measured in K-band, using normal modulation, with the flip-flop method. Once the loop has converged, the flip-flop modulation method has a consistently higher short exposure Strehl than the standard modulated pyramid. The resulting long exposure point spread function (PSF)s are shown in Fig. 18, where an improvement in the PSF core (first four rings) is visible with the flip-flop method.

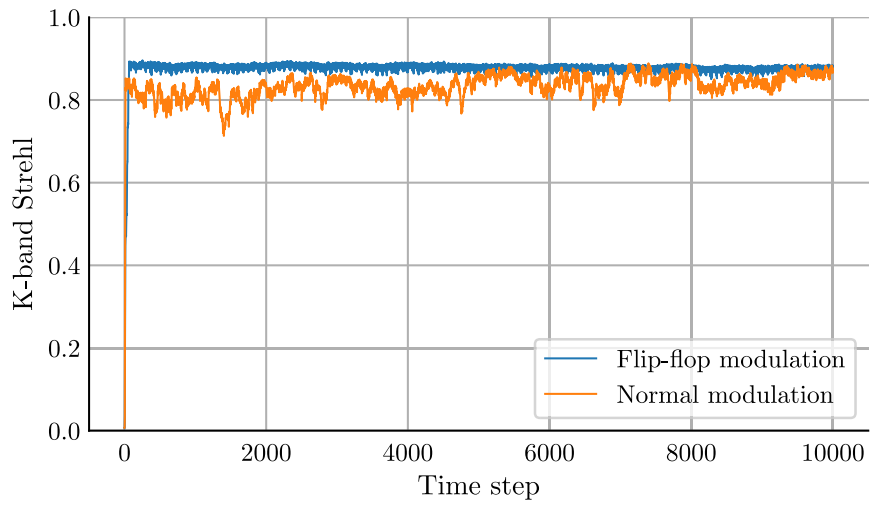

Fig. 17 The short exposure Strehl, measured in K-band, comparing the flip-flop modulation method (blue) with the traditional modulation method (orange).

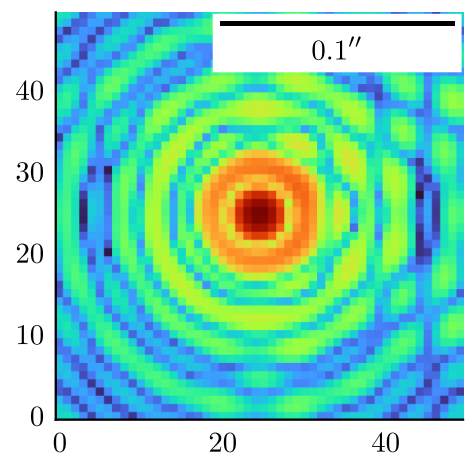

(a)

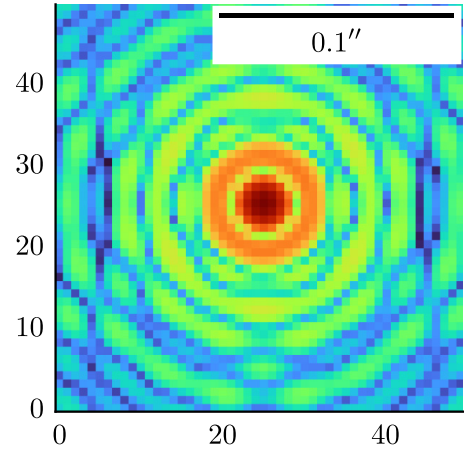

(b)

Fig. 18 The long exposure PSF simulated in K-band, with the WFS also operating in K-band, (a) without and (b) with the flip-flop modulation. 


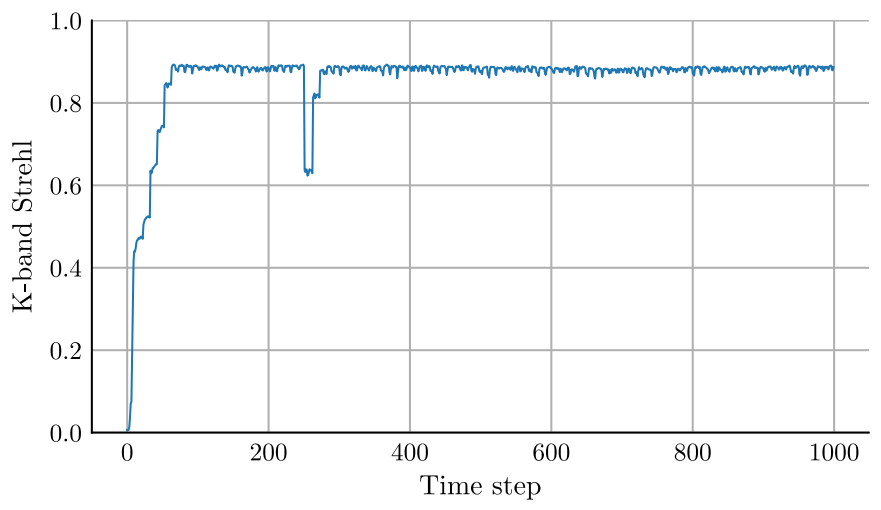

Fig. 19 The short exposure Strehl, simulated in K-band, using the flip-flop modulation method and the simulation parameters from Table 1. From time step 250, a segment piston waffle mode of $400-$ $\mathrm{nm}$ peak-to-valley is applied to the incoming wavefront.

The flip-flop modulation method is able to sense and correct large (400-nm peak-to-valley) segment piston errors in the atmospheric wavefront. Figure 19 shows the short exposure Strehl, measured in K-band, using the flip-flop method with a 400-nm peak-to-valley segment piston waffle (an alternating push and pull of the six segments) mode injected into the atmospheric phase from iteration 250. The short exposure Strehl returns to the baseline level within 25 iterations.

\subsection{R-Band Scenario}

In R-band, if the petal modes are not controlled, the closed-loop performance is poor, due to excessive petal piston. Figure 20 shows that using our flip-flop modulation approach, we are able to sense and correct petal modes, resulting in stable closed-loop performance with a closed-loop Strehl of $19.1 \%$ (Table 2). We are also able to sense and correct petal piston errors which are artificially added to the atmospheric phase.

The Maréchal criterion approximates the Strehl ratio

$$
\operatorname{Strehl} \approx e^{-\left(\frac{2 \pi \varepsilon}{\lambda_{p}}\right)^{2}},
$$

where $\epsilon$ is the RMS wavefront error in metres and $\lambda_{p}$ is the wavelength in metres. By taking an atmospheric phase screen and removing the projection of each of the 3500 modes in the KL modal basis including tip and tilt (before it has been forced orthogonal to segment piston eigenmodes), the RMS fitting error, $\epsilon$, is $\sim 110 \mathrm{~nm}$, resulting in a Strehl of $32.3 \%$. This Strehl only includes fitting error and represents the best case closed-loop performance, before accounting for temporal effects, giving an upper bound for the closed-loop Strehl in R-band. From the results in Table 2, the flip-flop modulation method achieves a closed-loop Strehl close to this fitting error, considering the fitting error does not account for any temporal errors.

In this method, the absolute position of each segment piston is not known; however, the segment piston errors are controlled relative to some offset ( $2 \pi$ ambiguity). In these monochromatic simulations, the segment piston errors were corrected, relative to some absolute offset. Not knowing what the value of the absolute offset is had no detrimental effect on the PSF as the wavefront sensing wavelength is the same as the PSF wavelength.

The long exposure PSFs are shown in Fig. 21, where using the flip-flop method an Airy disk is visible and the six diffraction spikes from the spider arms are just visible, unlike the PSF from the standard modulated scheme, where an Airy disk is not visible and the diffraction spikes are less prominent. 


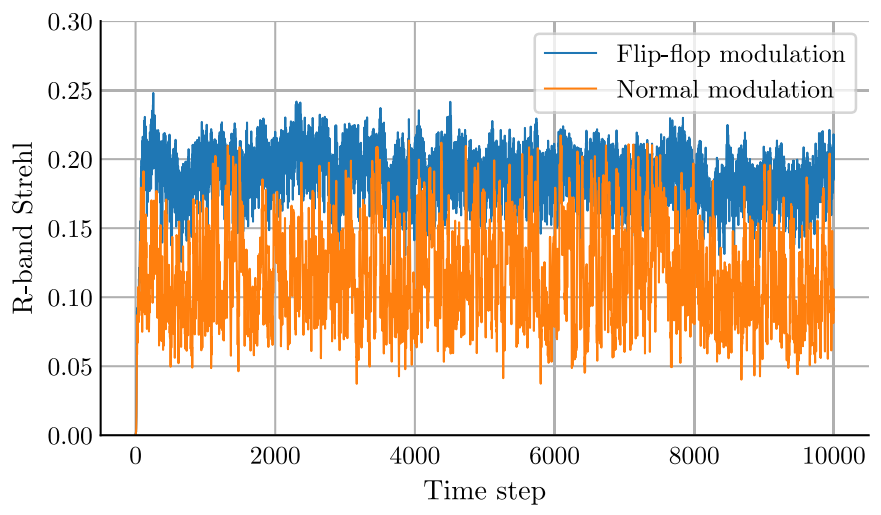

Fig. 20 The short exposure Strehl, measured in R-band, comparing the flip-flop modulation method (blue) with the traditional modulation method (orange).

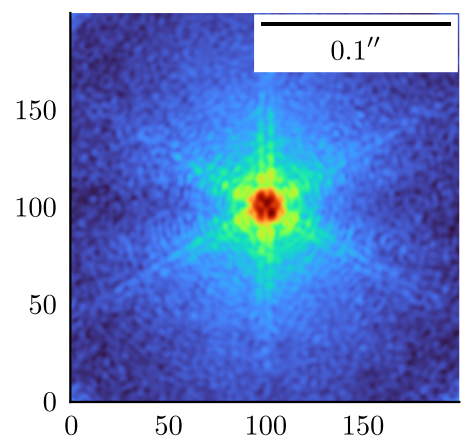

(a)

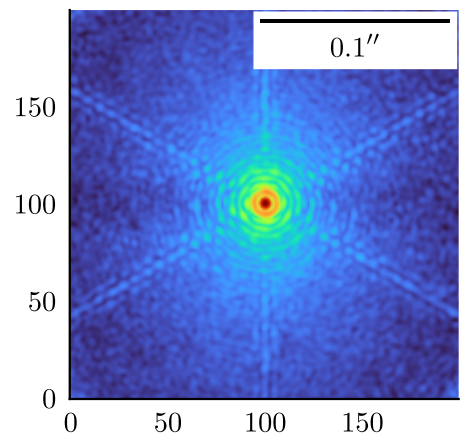

(b)

Fig. 21 The long exposure PSF simulated in R-band, with the WFS also operating in R-band, (a) without and (b) with the flip-flop modulation.

\section{Conclusion}

We have presented a new flip-flop modulation method for controlling segment piston errors that] are within a range of one wavelength, which is shown working in simulation, in both R- and Kbands with an $r_{0}$ of $15 \mathrm{~cm}$. The improvement of the flip-flop method versus a standard modulated pyramid WFS, in long exposure Strehl for K- and R-bands after 10,000 iterations, is 9.9\% and $13.0 \%$, respectively. The flip-flop modulation method introduces several new parameters that could be further optimized, such as the duty cycle and frequency of the unmodulated pyramid WFS loop (effectively controlling the bandwidth of the segment piston corrections).

In future work, we will evaluate the feasibility of stopping the modulation in one frame. If one frame is not sufficient, more dead-frames can be added but at a loss in segment piston control bandwidth.

The simulations in this paper only cover the monochromatic case, where a $2 \pi$ phase ambiguity has no detrimental effect on the PSF. In future work, it will be interesting to explore the flip-flop method with polychromatic light sources as well as using the continuous modal basis proposed by Bertrou-Cantou et al. ${ }^{14}$ for the modulated loop. Adding to the optical gain corrections of the reference signal of the pyramid, implementing a filter for the pyramid WFS measurement signal, which is matched to the segment piston eigenmode measurement signal, could further increase the signal-to-noise ratio.

\section{References}

1. R. Tamai et al., "The ESO's ELT construction progress," Proc. SPIE 11445, 114451E (2020) 
Engler et al.: Flip-flop modulation method used with a pyramid wavefront sensor to correct piston...

2. A. H. Bouchez et al., "An overview and status of GMT active and adaptive optics," Proc. SPIE 10703, 107030W (2018).

3. C. Boyer and B. Ellerbroek, "Adaptive optics program update at TMT," Proc. SPIE 9909, 990908 (2016).

4. R. Davies and M. Kasper, "Adaptive optics for astronomy," Annu. Rev. Astron. Astrophys. 50(1), 305-351 (2012).

5. P. Hickson, "Atmospheric and adaptive optics," Astron. Astrophys. Rev. 22, 76 (2014).

6. R. Ragazzoni, "Pupil plane wavefront sensing with an oscillating prism," J. Mod. Opt. 43(2), 289-293 (1996).

7. C. Vérinaud, "On the nature of the measurements provided by a pyramid wave-front sensor," Opt. Commun. 233(1), 27-38 (2004).

8. S. Esposito and A. Riccardi, "Pyramid wavefront sensor behavior in partial correction adaptive optics," Astron. Astrophys. 369, L9-L12 (2001).

9. T. Y. Chew, R. M. Clare, and R. G. Lane, "A comparison of the Shack-Hartmann and pyramid wavefront sensors," Opt. Commun. 268, 189-195 (2006).

10. R. Clare and M. Le Louarn, "Numerical simulations of an extreme AO system for an ELT," in 2nd Int. Conf. Adapt. Opt. for Extremely Large Telesc., Vol. 72 (2011).

11. N. Schwartz et al., "Sensing and control of segmented mirrors with a pyramid wavefront sensor in the presence of spiders," in Adapt. Opt. Extreamly Large Telesc., Vol. 5 (2017).

12. R. Holzlöhner et al., "Bolometric night sky temperature and subcooling of telescope structures," Astron. Astrophys. 645, A32 (2021).

13. V. Hutterer et al., "Advanced wavefront reconstruction methods for segmented Extremely Large Telescope pupils using pyramid sensors," J. Astron. Telesc. Instrum. Syst. 4(4), 049005 (2018).

14. A. Bertrou-Cantou et al., "Petalometry for the ELT: dealing with the wavefront discontinuities induced by the telescope spider," Proc. SPIE 11448, 1144812 (2020).

15. B. Engler et al., "Effects of the telescope spider on extreme adaptive optics systems with pyramid wavefront sensors," Proc. SPIE 10703, 107035F (2018).

16. S. Esposito et al., "Wavefront sensor design for the GMT natural guide star AO system," Proc. SPIE 8447, 84471L (2012).

17. B. Engler et al., "Pyramid wavefront sensing in the presence of thick spiders," in AO4ELT6 (2019).

18. V. Korkiakoski, C. Vérinaud, and M. L. Louarn, "Improving the performance of a pyramid wavefront sensor with modal sensitivity compensation," Appl. Opt. 47, 79-87 (2008).

19. V. Deo et al., "A modal approach to optical gain compensation for the pyramid wavefront sensor," Proc. SPIE 10703, 1070320 (2018).

20. V. Chambouleyron et al., "Pyramid wavefront sensor optical gains compensation using a convolutional model," Astron. Astrophys. 644, A6 (2020).

21. O. Lai, P. J. Stomski, Jr, and E. Gendron, "MANO: the modal analysis and noise optimization program for the W.M. Keck Observatory adaptive optics system," Proc. SPIE 4007, 620-631 (2000).

22. E. Gendron, "Optimization of modal control in adaptive optics: applications à l'astronomy," Theses, Université Denis Diderot (Paris 7) (1995).

23. M. Le Louarn et al., "Parallel simulation tools for AO on ELTs," Proc. SPIE 5490, 705-712 (2004).

24. S. Hippler et al., "Single conjugate adaptive optics for the ELT instrument METIS," Exp. Astron. 47, 65-105 (2019).

Biographies of the authors are not available. 